\title{
A Versatile Ultra-Stable Platform for Optical Multidimensional Fourier-Transform Spectroscopy
}

\author{
A. D. Bristow, D. Karaiskaj, X. Dai, T. Zhang, \\ C. Carlsson, K. R. Hagen, R. Jimenez and S. T. Cundifff \\ JILA, University of Colorado $\&$ National Institute of Standards and Technology, \\ Boulder, Colorado 80309-0440, USA
}

(Dated: October 31, 2018)

\begin{abstract}
The JILA Multidimensional Optical Nonlinear SpecTRometer (JILA-MONSTR) is a robust, ultra-stable platform consisting nested and folded Michelson interferometers that can be actively phase stabilized. This platform generates a square of identical laser pulses that can be adjusted to have arbitrary time delay between them, while maintaining phase stability. The JILA-MONSTR provides output pulses for nonlinear excitation of materials and phase-stabilized reference pulses for heterodyne detection of the induced signal. This arrangement is ideal for performing coherent optical experiments, such as multidimensional Fourier-transform spectroscopy, which records the phase of the nonlinear signal as a function of the time delay between several of the excitation pulses. The resulting multidimensional spectrum is obtained from a Fourier transform. This spectrum can resolve, separate and isolate coherent contributions to the light-matter interactions associated with electronic excitation at optical frequencies. To show the versatility of the JILA-MONSTR, several demonstrations of two-dimensional Fourier-transform spectroscopy are presented, including an example of a phase-cycling scheme that reduces noise. Also shown is a spectrum that accesses two-quantum coherences, where all excitation pulses require phase locking for detection of the signal.
\end{abstract}




\section{INTRODUCTION}

Multidimensional Fourier-transform spectroscopy is an important tool for elucidating structure and dynamics of matter. ${ }^{1]}$ It was developed at radio frequencies using a sequence of electromagnetic pulses, although it is applicable to any region of the electromagnetic spectrum where there exists the ability to generate, manipulate and detect pulsed radiation. Over

the last decade, multidimensional concepts have been applied in optical experiments, ${ }^{[2}$ where short intense pulses are generated by lasers. In the infrared, these techniques can explore vibronic resonances to determine the structure and coherent dynamics of molecules ${ }^{3-6}$ At higher optical frequencies the light excites and probes electronic transitions in molecules 78 and semiconductors. $\frac{9 \mid 10}{9}$

Multidimensional Fourier-transform spectroscopy explicitly tracks the phase of an induced nonlinear signal generated by the sequence of excitation pulses ${ }^{11}$ Tracking the phase with respect to the time delays between excitation pulses results in a multidimensional time-domain data set, which is converted to a multidimensional spectrum with a Fourier-transform. This technique has several advantages over other electromagnetically driven spectroscopic techniques: the phase is tracked explicitly, capturing the induced complex polarization in the sample; coherent coupling between states is consequently resolved; the nonlinear response is unfolded onto a multidimensional plane, thus separating and isolating otherwise overlapping spectral contributions in the response; and coherent non-radiative contributions can also be isolated with the appropriate time-ordering of the excitation pulses.

Numerous methods exist for implementing optical multidimensional Fourier-transform spectroscopy, all of which require sub-wavelength stability. These methods can include passive stabilization, such as sturdy construction and common paths through optical elements, $\frac{12}{14}$ or active stabilization that employs feedback loops to suppress the effects mechanical drift 15 Previously published schemes include combinations of diffractive optics elements to create spatially separated pulses, $\frac{12 \mid 13}{2}$ and pulse shapers to vary the time and

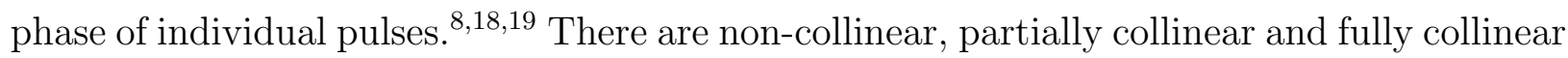
implementations of 2DFT spectroscopy, the latter of which require phase cycling to extract the signal. ${ }^{8 / 20121}$ Most commonly employed is the background-free transient four-wave mixing (TFWM) geometry 12 14]16 18

In this paper, we present an ultra-stable platform that provides four identical laser pulses 
in a non-collinear box geometry. This platform is designed for optical multidimensional Fourier-transform spectroscopy, and has been dubbed the "JILA Multidimensional Optical Nonlinear SpecTRometer" (JILA-MONSTR). The JILA-MONSTR is based on a threepulse TFWM excitation scheme, using the fourth pulse as a phase-stable reference pulse. The versatility of the platform is demonstrated with several 2DFT projections, including a technique that extracts two-quantum coherent and requires all excitation pulses to be phase locked. Also presented is an optional, straight forward implementation of phase cycling to reduce noise from scattered pump light in the non-collinear geometry. ${ }^{22}$ Note that all-optical phase retrieval is not discussed because extensive details are found in Ref. 23. Semiconductor quantum wells $s^{24 \mid 25}$ and potassium vapor ${ }^{26}$ are used to demonstrate the regular and phasecycling operation of the JILA-MONSTR, respectively.

\section{EXPERIMENTAL}

\section{A. Excitation Scheme}

The JILA-MONSTR is based on a three-pulse excitation scheme, which enables the acquisition of one-, two- and three-dimensional spectra. The excitation pulses are assigned the notation $\mathrm{A}^{*}, \mathrm{~B}$ and $\mathrm{C}$, where pulse $\mathrm{A}^{*}$ is phase conjugated with respect to pulses $\mathrm{B}$ and C. In the non-collinear box geometry, the excitation pulses lie on three corners of a square. The TFWM signal therefore has a phase-matching condition $\left(k_{s i g}\right)$ that is some permutation of the excitation wavevectors $-k_{A}, k_{B}$ and $k_{C}$. Any permutation of the phase-matching condition can be measured by changing the time ordering of the pulses, without altering the signal collection path. Note that two-pulse excitation schemes can also be recorded in a different direction that require a change in the signal collection path.

Figure 1 shows the possible pulse sequences available with three-pulse excitation. The pulse ordering is germane to the dephasing information induced in the excited material. In Fig. 1(a) the first and third pulses are phase conjugated with respect to one another. This excitation sequence undoes the effect of sample inhomogeneity, leading to a photon echo. Hence, this pulse sequence is referred to as either $S_{I}$ or "rephasing." In this case, because the first and third time axes are interrogated, the Fourier transform gives a spectrum with

respect to $\omega_{\tau}$ and $\omega_{t}$ (see inset). Figure $1(\mathrm{~b})$ is a variant of the rephasing projection where 
the Fourier transform is with respect to time axes $T$ and $t$, allowing the non-radiative contributions of the second time period to be explored. ${ }^{27}$ In Fig. 1(c) the conjugate pulse is second in the sequence and, unlike Fig. 1(a), the inhomogeneity is not cancelled leading to a free decay. This scheme is therefore referred to as either $S_{I I}$ or "non-rephasing." Similarly, in Fig. 1(d) rephasing does not occur because the conjugate pulse is third in the sequence.

Hence, this sequence is known as $S_{I I I}$. Since the first two pulses are not phase conjugates of one another they can coherently add allowing access to two-quantum information. Hence, the second and third time periods are Fourier transformed to yield the 2DFT spectrum.

This scheme requires three non-collinear excitation pulses and a heterodyne reference (Ref) and/or tracer (Tr) pulse. All pulses must be controlled with sub-cycle precision over delay ranges that are large compared to the coherence length of the TFWM emission in most materials. This requirement can be achieved using mechanical translation stages and sturdy apparatus construction, enhanced by active feedback electronics. Phase control between any two pulses can be achieved using the error signal of a co-propagating continuous-wave (CW) laser beam in a Michelson interferometer. In which case, the error signal is the interfering term between the superposition of the CW light in each arm of the interferometer. Phase control of four pulses can be achieved with a set of nested interferometers; see the schematic diagram shown in Fig. 2. In this setup, the pulses exit through the dichroic mirrors (DCMs) and the CW laser is reflected, producing error signals at the diagnostic ports. Error signals are recorded and fed into electronic loop filters that adjust piezo-electric transducers (PZTs) in each interferometer (not shown in this diagram). Several delay stages are placed in convenient locations within the setup. To create the box of output pulses this system must be folded.

\section{B. The JILA-MONSTR}

The scheme presented above is implemented in the JILA-MONSTR, which comprises two decks with the optics for an interferometer on each deck and an additional interferometer between the decks.28 Computer-aided design drawings of the lower and upper decks are shown in Fig. 3(a) and (b), respectively. The decks are made from cast aluminum and have a mass of $39 \mathrm{~kg}$ each. This material avoids both longer term relaxation of rolled metal slabs and reduces shorter term thermal drift. Each deck is milled, using computer numerical 
control software, to house the optics for each interferometer. The lower deck supports the interferometer for pulses $\mathrm{C}$ and Ref; the long delay stage (U), which moves $\mathrm{C}$ and Ref together with respect to the top deck pulses; the PZT for the bottom-deck interferometer; and the short delay stage $(\mathrm{Z})$, which changes the time delay between pulses $\mathrm{C}$ and Ref. The top deck supports the interferometer for pulses $\mathrm{A}^{*}$ and $\mathrm{B}$; the PZTs to control the phase of the top- and inter-deck interferometers; and two short delay stages (X and Y), which allow arbitrary variation of time position of pulse $\mathrm{A}^{*}$ and $\mathrm{B}$. This platform has a total of four direct-drive linear translation stages with sub-nanometer resolution: X,Y,Z stages have $5 \mathrm{~cm}$ of travel and the $\mathrm{U}$ stage has $20 \mathrm{~cm}$ of travel.

The decks and all optical mounts are custom made, except for two sturdy three-axis, kinematic mirror mounts. All mirrors are coated with protected Ag for operation close to $800 \mathrm{~nm}$. Three broadband beamsplitters (BS) divide the input laser pulse into four replicas. The BS coatings are centered at $800 \mathrm{~nm}$, on thin and low group-velocity dispersion substrates. Compensation plates $(\mathrm{CP})$, made from identical substrates, balance the dispersion in the appropriate arm of each interferometer. All transparent and semitransparent optics are antireflection coated, and mounted in strain-minimizing mounts with o-rings to prevent induced birefringence.

The decks form the exoskeleton of the JILA-MONSTR, enveloping the optics in a 5 -cm gap; see Fig. 3(c). Five mounting points ensure a sturdy enclosure. The small gap reduces exposure of the optics to air currents, and they are further protected with side panels. After assembly the 5-cm DCM is attached to the front of the JILA-MONSTR, reflecting the CW laser light back into the device and completing the interferometers. The interferometric error signals are shown as dashed lines emerging from the right-hand side of the JILA-MONSTR (denoted $\mathrm{R}$ in the figure). The laser pulses that perform the 2DFT experiment emerge through the DCM in a square that is $5 \mathrm{~cm}$ along each side. Figure 4 shows a photograph of the closed JILA-MONSTR with the input and output beams highlighted.

Alignment of the JILA-MONSTR starts with the separated decks, individually prior to assembly. Each deck must provide two parallel output beams, that do not deviate when the delay stages are scanned, while producing clean interference patterns at the diagnostic port. (Only one interferometer works per deck before closing the assembly). When the assembly is closed, the folded interferometers are completed by the single 5-cm DCM, which requires accurate alignment to optimize all three interferometeric error signals at the diagnostic port. 
Consequently, the JILA-MONSTR has been designed such that excess degrees of freedom are remove from the optical paths. Two quadrant-diode photodetectors (QD) assist with daily optimization of the alignment.

Figure 5(a) shows the error signals of the three interferometers of the JILA-MONSTR, recorded at the diagnostic port with the active feedback loops disengaged. The path length of the interferometer arms drift over several minutes, leading to changes in phase of $>2 \pi$. In contrast, the error signals are held at zero volts when active stabilization is engaged; see Fig. 5(b). Note that the bottom-deck and inter-deck error signals are offset by $1 \mathrm{~V}$. The variations of the locked error signals are normal distributions, with a standard deviation that corresponds to $\sim 2 \mathrm{~nm}$ of motion. This motion corresponds to phase stabilization of $\lambda / 130$ up to $\lambda / 400$, assuming that the laser pulses for the 2DFT experiment have a wavelength of $800 \mathrm{~nm}$.

To perform a 2FDT scan, an individual time delay must be moved many 100's of times with perfectly spaced increments. Each of these increments is computer controlled. This control process is discussed in more detail in the next subsection, after the introduction of the setup downstream from the JILA-MONSTR.

\section{Setup}

Figure 6 shows the entire experimental setup for multidimensional spectroscopy. The pulsed laser source is a mode-locked Ti:sapphire oscillator. It operates at $76 \mathrm{MHz}$, producing $\sim 100$ fs pulses that are tuned near $800 \mathrm{~nm}$ or to $768 \mathrm{~nm}$ for the semiconductor or potassium vapor experiments, respectively. The CW stabilization source is a HeNe laser operating at $632.8 \mathrm{~nm}$. These are combined on a DCM situated before the entrance to the JILAMONSTR.

The pulses from the JILA-MONSTR are focused by a single lens to overlap at the sample under investigation, as shown in the photograph (Fig.4) and schematic diagram (Fig. 6). Three of the pulses are excitation pulses, generating the TFWM signal. The fourth pulse is attenuated and is used as the heterodyne reference pulse. Note that for many 2DFT experiments the reference (local oscillator) transmits through the sample. In some cases however, the nonlinear signal is too sensitive to the excitation conditions. Hence, a phasestabilized reference is routed around the sample and recombined with the signal further 
downstream (details not shown in Fig. 6)! $\frac{1723}{\sqrt{123}}$ In either case, the signal and reference pulses propagate collinearly to the spectrometer, temporally delayed by several picoseconds. A spectral interferogram is captured by the spectrometer (see inset of Fig. 6), from which the complex spectrum is obtained 29

A 2DFT spectrum is constructed by incrementally adjusting one time delay while recording spectral interferograms. The complete set of data is converted to a two-dimensional spectrum by a one-dimensional numerical Fourier transform along the direction of the scanned time axis. The incremental adjustment and acquisition of the 2DFT spectrum is automated, as shown in the flow chart in Fig. 7(a). The sequence of events is: the feedback loops of the appropriate interferometers are disengaged; the desired translation stage(s) are moved a specific distance; after a short time, allowing the delay stage to reach its new position, the feedback loops are reengaged; and a spectral interferogram is recorded. This procedure is the main loop of the 2DFT acquisition algorithm, and is repeated until the length of the scan is sufficient to collect the full dephasing of the TFWM signal. Within the main loop, the locked and unlocked error signals are recorded every time the feedback loops are disengaged. The recorded values are used to recalculate the distance to move the delay stage for that step. Repeating this calculation every step ensure that the feedback loops are never forced to the extremes of their compensation range, and speeds up the scan rate. Even though a timeout can occur before the feedback loops are reengaged, the scan is sufficiently stable that only a catastrophic electronics glitch cannot be corrected. While scan rates are high, continuous scanning methods can potentially improve acquisition rates to shorten the overall acquisition time.

In addition to recording the error signals and PZT voltages, the experimenter can monitor the error signals in real time on a a $100 \mathrm{MHz}$, 4-channel oscilloscope. Figure 7(b) shows a typical screen capture of one error signal during a single step of distance commensurate with 4 HeNe fringe. (Nyquist undersampling is used during the data analysis to correctly determine the frequency of the 2DFT signal after the Fourier transform. 17) The fast oscillations are due to the motion of the stage, when the feedback loop is disengaged. The shaded regions show when the feedback loops are engaged while the spectral interferogram is recorded.

Two samples are used to demonstrate the versatility of the JILA-MONSTR. Firstly, data is shown from 4-period multiple quantum well sample grown by molecular beam epitaxy. The semiconductor sample consisting of $10 \mathrm{~nm}$ GaAs wells and $\mathrm{Al}_{0.3} \mathrm{Ga}_{0.7} \mathrm{As}$ barriers, with 
an etch stop layer that allows chemical removal of the thick GaAs substrate. Measurements are performed in a cryostat, cooled to $\sim 6 \mathrm{~K}$. Secondly, results are presented from a thin potassium vapor cell, where the potassium is heated to $523 \mathrm{~K}\left(250{ }^{\circ} \mathrm{C}\right)$. The thickness of the cell is approximately $6 \mu \mathrm{m}$. In both samples the effective optical thickness is chosen to minimize reabsorption of the induced radiation.

\section{RESULTS}

The JILA-MONSTR is capable of performing one-dimensional linear and non-linear spectroscopy as well as two- and three-dimensional non-linear spectroscopy. Versatility arises from the pulse layout. Single-pulse linear measurements can be performed to obtain the absorption or photoluminescence. Two-pulse nonlinear pump-probe measurements can also be performed using the attenuated tracer pulse as a probe and any one of the other pulses as a strong pump. Three-pulse excitation results in TFWM signals and higher-dimensional Fourier-transform spectroscopy. In this section, 2DFT results are presented with references to related work.

Linear absorbance for the quantum well sample is shown in the top panels of Fig. 8. Two resonances are observed, which are associated with bound electron-hole pairs, or exciton states. The lower and higher energy absorption peaks are the heavy-hole $\left(X_{h h}\right)$ and lighthole $\left(X_{l h}\right)$ excitons, respectively. Increased absorption on the higher energy side of $X_{l h}$ is due to absorption from the heavy-hole valence band to the continuum conduction states at energies above the discrete states of the quantum wells. The binding energy of the $X_{h h}$ is approximately $8 \mathrm{meV}$, which is similar to the $X_{h h}$ and $X_{l h}$ separation in this sample.

\section{A. 2DFT Spectra}

The complexity of the coherent response of semiconductor quantum wells lends itself to study by 2DFT spectroscopy, because this technique separates many of the competing processes. Figure 8 (b,c and e) shows the real part of the rephasing, non-rephasing and twoquantum 2DFT spectra. The emission axis defines the numerical sign of the photon energies. Due to the location of pulse $A^{*}$ in the excitation sequence, the absorption energy is in the positive (negative) quadrant for non-rephasing (rephasing). These spectra are normalized 
to their respective strongest features.

Spectral features that appear on the diagonal (in the single-quantum spectra) and doublediagonal (in the two-quantum spectrum) are coherent intra-action of the $X_{h h}$ or $X_{l h}$. Offdiagonal features correspond to coherent interaction between these states. The coherent response of the quantum well is very sensitive to the excitation conditions, and the spectral features reveal the presence of many-body interactions. 30131 Additionally, elongation of features in the rephasing 2DFT spectra arises from inhomogeneous broadening due to well width fluctuations. ${ }^{32}$ Fig. 8 (b) and (c) are acquired with co-circularly polarized pump pulses, which excludes the formation of biexcitons. Biexcitons are observed for other polarization

configurations and their 2DFT spectroscopic fingerprint have been discussed recently $[3133$ Biexciton contributions are also observed in the two-quantum 2DFT spectra. $34 \sqrt[35]{3}$

The coherent two-quantum transition in semiconductor quantum wells have been previously studied with TFWM $\stackrel{36}{36}$ However, these experiments have left many unresolved questions. Excitation of two-quantum coherences requires that all excitation pulses be phase locked, $\stackrel{37 / 38}{ }$ and the first two pulses (in a three-pulse scheme) must not be phase conjugated with respect to one another. For $S_{I I I}\left(\tau, \omega_{T}, \omega_{t}\right)$ the pulse sequence is B then $\mathrm{C}$ followed by $A^{*}$; see Fig. 1(d). The final pulse then examines coherent transitions from the two-quantum to one-quantum states.

Figure $8(\mathrm{~d})$ shows the linear absorption for the quantum well sample. The excitation laser is tuned over the $X_{h h}$. Figure 8(e) shows an example of the real part of 2DFT spectrum of GaAs quantum wells using the two-quantum technique, where $\tau=0$ fs and the polarization configuration is cross-circular. The two-quantum spectrum is plotted as a function of twoquantum absorption energy $\left(2 \hbar \omega_{T}\right)$ versus the emission photon energy $\left(\hbar \omega_{t}\right)$. The dashed line marks the double-diagonal. In the $S_{I I I}$ spectrum the two-quantum contributions are many-body scattering states. 34

\section{B. Phase Cycling and Noise Reduction}

Phase cycling has been used in multidimensional $\mathrm{NMR}^{1}$ and collinear optical 2DFT spectroscopy $\stackrel{820121}{10}$ to extract signals and remove noise, interference effects or multiplequantum contributions. It is therefore useful for non-collinear experiments ${ }^{22}$ that suffer from a low signal-to-noise ratio. Some situations may be dominated by noise sources such 
as pump scatter, which emanates from the same location as the TFWM signal. In 2DFT spectra, pump scatter is observed along the diagonal of the single-quantum spectra, because the pump is only self-coherent, i.e. only correlated when $\omega_{\tau}=\omega_{t}$. This is not an issue for two-quantum spectra, because the Fourier transform yields a different spectral range, without pump scatter. In the single-quantum 2DFT spectra, cycling the phase of the excitation pulses during a 2DFT scan suppresses the noise along the diagonal.

In NMR spectroscopy phase alternative pulse sequences (PAPS) are used, where the relative phase of the two RF pulses switches between in phase and out of phase every time step in a scan. Incorrectly phased artifacts in the detected signal then cancel when the Fourier transform is performed, leaving only the signal in the two-dimensional spectrum. The optical analogy of PAPS employed here records and averages two spectral interferograms that have nearly identical time delay and a controlled phase shift between two pairs of beams, thus allowing the incorrectly phased noise to cancel when averaged. The example used here is for an adjustment of the second time period $T$, such that the phase that is cycles is proportional to $\phi_{T}=n \pi+\omega_{T} T$, where the first term is toggled between $0 \pi$ and $1 \pi$ for every time step along axis $\tau$. Figure 9 (a) shows how the phase-cycling scheme is implemented in the four pulses excitation sequence of the JILA-MONSTR. The phase between the first two pulses ( $\mathrm{A}^{*}$ and $\mathrm{B}$ ) and the second two pulses ( $\mathrm{C}$ and Ref) are shifted. In the JILAMONSTR, this is achieved by moving the long delay stage (U) back and forth for every incremental position of the first excitation pulse; see the inset of Fig. 9(b). The motion uses the same disengage-move-reengage algorithm shown in the main loop of Fig. 6(a). In practice the wavelengths of the excitation and phase-control lasers are incommensurate: the example presented here is performed on the $\mathrm{D}_{1}$ and $\mathrm{D}_{2}$ lines of potassium vapor; thus, the Ti:sapphire excitation wavelength is $768 \mathrm{~nm}$. The most convenient step size of the $\mathrm{U}$ stage is therefore 6 HeNe fringes, which corresponds to approximately $5 \pi$ at the Ti:sapphire wavelength.

Figure 9(b) shows an example of the TFWM signal plotted along the reconstructed emission time $t$, with and without phase cycling. The reconstructed axis is obtained by the numerical Fourier transform of the heterodyne-detected interferogram. In these transient the time delays $\tau$ and $T$ are set to 1 ps. The dashed transient is acquired for normal operation of the JILA-MONSTR, and thus constructed from a single interferogram at $0 \pi$. In contrast, the averaging of the phase-cycled spectral interferograms is shown as a solid line, where 
contributions from $\mathrm{A}^{*}$ and $\mathrm{B}$ are suppressed.

Figure 10 shows 2DFT spectra for both normal and phase-cycling operation of the JILAMONSTR. The top (bottom) row shows non-rephasing (rephasing) data, and the left column is acquired without phase cycling, where diagonal streaks are seen in both spectra. These diagonal streaks are suppressed in the right-hand side of the figure, showing the advantage of phase cycling and the rapid temporal flexibility of the JILA-MONSTR. Note that more complex phase-cycling schemes can be employed to remove the effect of all pump pulses.

\section{CONCLUSION}

In summary, we have shown a versatile platform for performing optical multidimensinoal Fourier-transform spectroscopy. The JILA-MONSTR uses an active stabilization scheme to provide four identical phase-locked pulses arranged in a box geometry. The active, computer-controllable feedback loops combined with electromechanical delay stages allow the phase-locked pulses to be ordered in any sequence with arbitrary time delays. The JILA-MONSTR has a stability of greater than $\lambda / 100$ and employs a stepping scheme that allows for acquisition of full 2DFT spectra, even for samples with long dephasing times.

The JILA-MONSTR can perform one-, two- and three-dimensional spectroscopy. It can be used for numerous excitation techniques within the scope of the various dimensions. Here we have demonstrated selected examples of $S_{I}, S_{I I}$ and $S_{I I I}$ techniques. In addition to the versatility, stability and speed of acquisition, the JILA-MONSTR offers straightforward implementation of phase-cycling to improving data quality. Here we have demonstrated a useful phase-cycling scheme to suppress pump scatter.

The JILA-MONSTR has proven to be a robust platform for producing multidimensional spectra. It is a viable way to separate and isolate the competing contributions to coherent light-matter interactions. This sophisticated device has great potential in the future of optical spectroscopy.

Acknowledgements: The authors thank Richard Mirin for the epitaxially grown quantum well sample. This work was supported by the National Science Foundation and the Chemical Sciences, Geosciences, and Biosciences Division Office of Basic Energy Sciences, U.S. 
Department of Energy.

* Electronic address: cundiffs@jila.colorado.edu

1 R. R. Ernst, G. Bodenhausen, A. Wokaun, "Principles of Nuclear Magnetic Resonance in One and Two Dimensions" (Oxford Univ. Press, Oxford, 1987).

2 M. Cho, Chem. Rev. 108, 1331 (2008).

3 P. Hamm, M. H. Lim, and R. M. Hochstrasser, J. Phys. Chem. B 102, 6123 (1998).

4 M. C. Asplund, M. T. Zanni and R. M. Hochstrasser, Proc. Natl. Acad. Sci. USA, 97, 8219 (2000).

5 O. Golonzka, M. Khalil, N. Demirdöven and A. Tokmakoff, Phys. Rev. Lett. 86, 2154 (2001).

6 S. Sul, D. Karaiskaj, Y. Jiang and N.-H. Ge, J. Phys. Chem. B 110, 19891 (2006).

7 J. D. Hylb, A. W. Albrecht, S. M. Gallagher Faeder and D. M. Jonas, Chem. Phys. Lett. 297, 307 (1998).

8 P. F. Tian, D. Keusters, Y. Suzaki and W. S. Warren, Science 300, 1553 (2003).

9 C. N. Borca, T. Zhang, X. Q. Li and S. T. Cundiff, Chem. Phys. Lett. 416, 311 (2005).

10 W. Langbein and B. Patton, Opt. Lett. 31, 1151 (2006).

11 S. Mukamel, Annu. Rev. Phys. Chem. 51, 691 (2000).

12 M. L. Cowan, J. P. Ogilvie, and R. J. D. Miller, Chem. Phys. Lett. 386, 184 (2004).

13 T. Brixner, I. V. Stiopkin, and G. R. Fleming, Opt. Lett. 29, 884 (2004).

14 U. Selig, F. Langhojer, F. Dimler, T. Löhrig, C. Schwarz, B. Gieseking and T. Brixner, Opt. Lett. 33, 2851 (2008).

15 L. Lepetit and M. Joffre, Opt. Lett. 21, 564 (1996).

16 V. Volkov, R. Schanz and P. Hamm, Opt. Lett. 30, 2010 (2005).

17 T. Zhang, C. N. Borca, X. Q. Li and S. T. Cundiff, Opt. Express 13, 7432 (2005).

18 J. C. Vaughan, T. Hornung, K. W. Stone, and K. A. Nelson, J. Phys. Chem. A 111, 4873 (2007).

19 E. M. Grumstrup, S. H. Shim, M. A. Montgomery, N. H. Damrauer, and M. T. Zanni, Opt. Express 15, 16681 (2007).

20 W. Wagner, C. Q. Li, J. Semmlow and W. S. Warren, Opt. Express 13, 3697 (2005).

21 P. F. Tekavec, G. A. Lott, and A. H. Marcus, J. Chem. Phys. 127, 214307 (2007). 
22 S. H. Shim, D. B. Strasfeld, Y. L. Ling, and M. T. Zanni, Proc. Natl. Acad. Sci. USA 104, 14197 (2007).

23 A. D. Bristow, D. Karaiskaj, X. Dai and S. T. Cundiff, Opt. Express 16, 18017 (2008).

24 V. M. Axt and T. Kuhn, Rep. Prog. Phys. 67, 433 (2004).

25 S. T. Cundiff, Opt. Express 16, 4639 (2008).

26 V. O. Lorenz, S. Mukamel, W. Zhuang and S. T. Cundiff, Phys. Rev. Lett. 100, 013603 (2008).

27 L. Yang, T. Zhang, A. D. Bristow, S. T. Cundiff and S. Mukamel, J. Chem. Phys. 129, 234711 (2008).

28 This design was inspired in part by a similar approach; see M.S. Pshenichnikov, "2D Optical Correlation Spectroscopy of Liquid/Glass Dynamics," 3rd International Conference on Coherent Multidimensional Spectroscoy, Rigi Kulm, Switzerland (2006).

29 L. Lepetit, G. Chériaux and M. Joffre, J. Opt. Soc. Am. B 12, 2467 (2005).

30 X. Q. Li, T. Zhang, C. N. Borca and S. T. Cundiff, Phys. Rev. Lett. 96, 057406 (2005).

31 T. Zhang, I. Kuznetsova, T. Meier, X. Q. Li, R. P. Mirin, P. Thomas and S. T. Cundiff, Proc. Natl. Acad. Sci. USA 104, 14227 (2007).

32 I. Kuznetsova, T. Meier, S. T. Cundiff and P. Thomas, Phys. Rev. B 76, 153301 (2007).

33 A. D. Bristow, D. Karaiskaj, X. Dai, R. P. Mirin and S. T. Cundiff, Phys. Rev. B 79, 161305(R) (2009).

34 S. T. Cundiff, T. Zhang, A. D. Bristow, D. Karaiskaj and X. Dai, Acc. Chem. Res. (2009), DOI:10.1021/ar9000636.

35 L. Yang and S. Mukamel, Phys. Rev. Lett. 100, 057402 (2008).

36 K. Ferrio and D. G. Steel, Phys. Rev. B 54, R5231 (1996).

37 K. W. Stone, K. Gundogdu, D. B. Turner, X. Li, S. T. Cundiff and K .A. Nelson, Science 324, $1169(2009)$.

38 D. Karaiskaj, A. D. Bristow, L. Yang, X. Dai, R. P. Mirin, S. Mukamel, S. T. Cundiff, submitted for publication, arXiv:0906.4068v1 [cond-mat.mes-hall] 


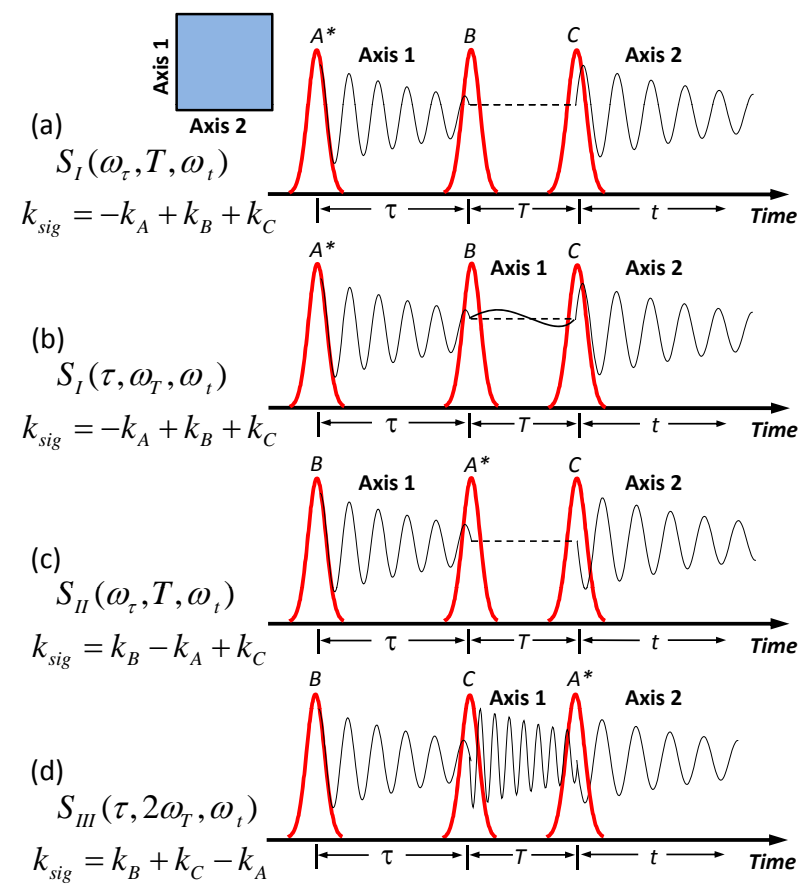

FIG. 1: (Color online) Schematic diagrams of the pulse ordering of optical 2DFT techniques available with a three-pulse excitation scheme: (a) represents the "rephasing" $S_{I}$ technique; (b) is a variant of (a) where the middle time axis is scanned to examine non-radiative contributions; (c) is "non-rephasing $S_{I I}$ technique; and (d) is the two-quantum $S_{I I I}$ technique. In each case Axis 1 and Axis 2 are labelled for comparison to the inset of (a) showing which axes are plotted in the resulting $2 \mathrm{DFT}$ spectrum. 


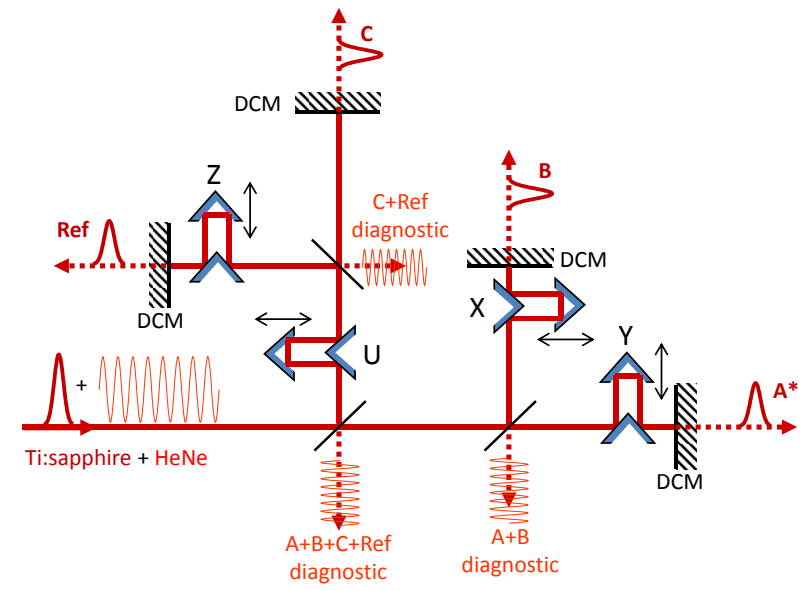

FIG. 2: (Color online) Schematic representation of the nested Michelson interferometer layout. A*, B, C and Ref are the Ti:sapphire laser pulses used to perform the 2DFT experiment. The HeNe laser beam is reflected from the DCM to provide diagnostic information for the interferometers. The proposed layout is folded such that the DCM is a common optical element. 

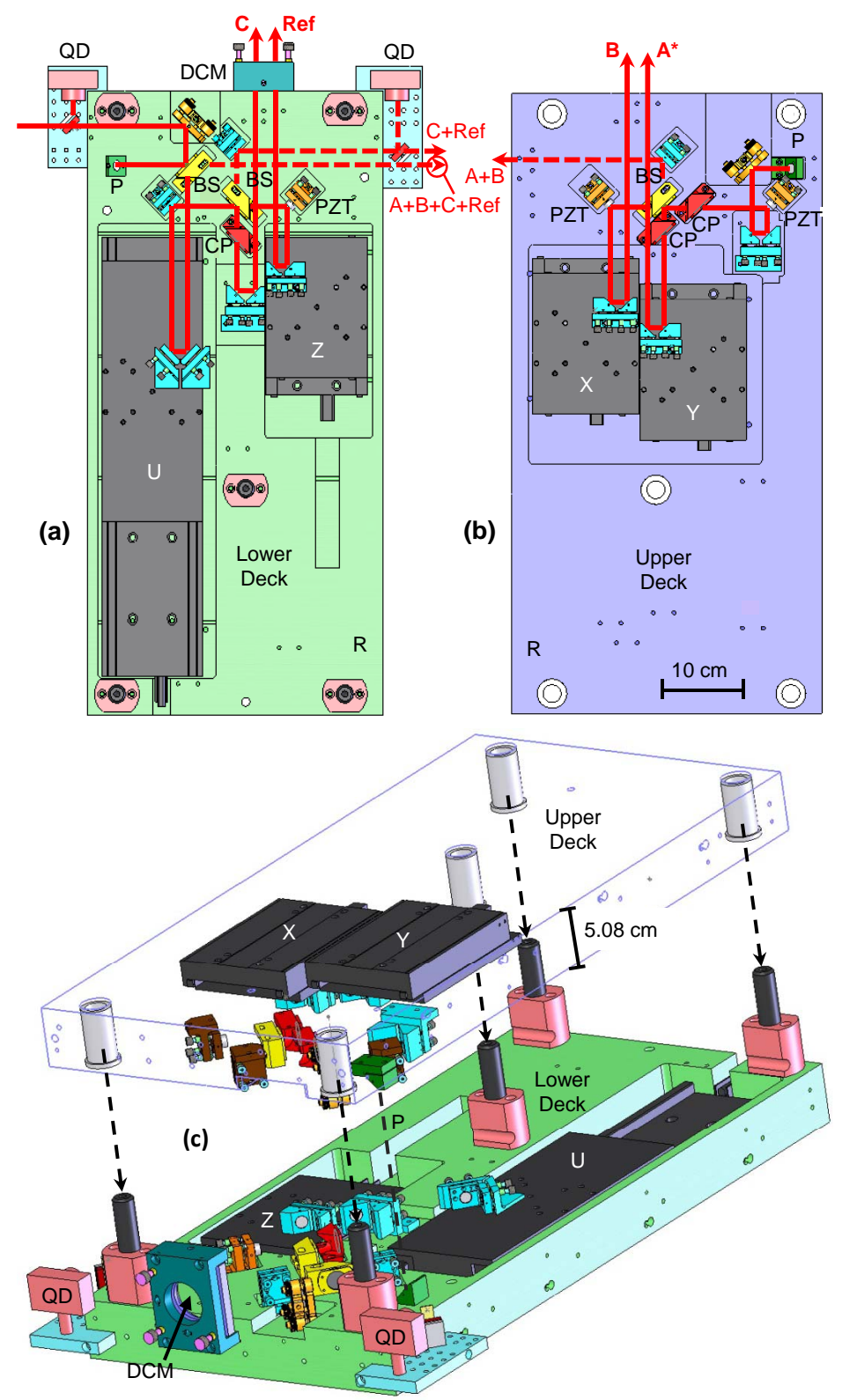

FIG. 3: (Color online) Computer Aided Design drawings of the JILA-MONSTR's (a) lower and (b) upper interferometer decks. P refers to the periscope between decks and $\mathrm{R}$ indicates the righthand side on each deck. Laser input is on the left side of the lower deck and the Ti:sapphire pulses emerge from the front through the dichroic mirror (DCM), two from each deck. Error signals for each interferometer emerge from the right-hand side as dashed lines, two from the bottom and one from the top decks. $[\mathrm{BS}=$ beamsplitter, $\mathrm{CP}=$ compensation plate, $\mathrm{PZT}=$ piezo-electric transducer, QD = quad-diode photodetector.] (c) Three-dimensional CAD drawings of the entire assembly showing how the two decks are assembled. 


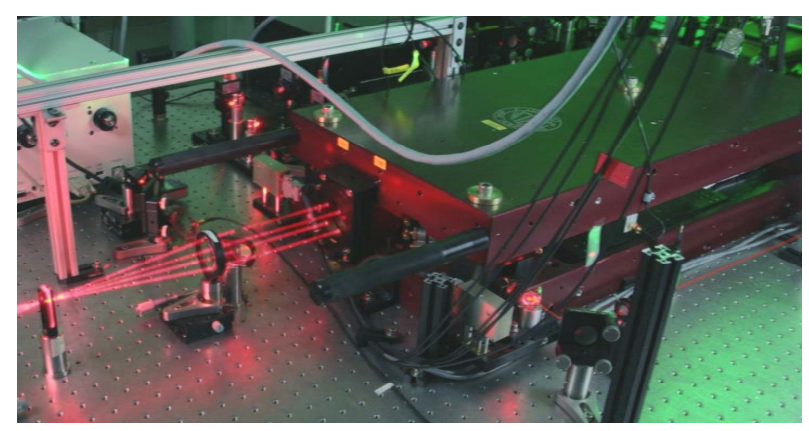

FIG. 4: (Color online) Photograph of the JILA-MONSTR highlighting the input beam on the right-hand side of the picture and four output beams focused to a single location.

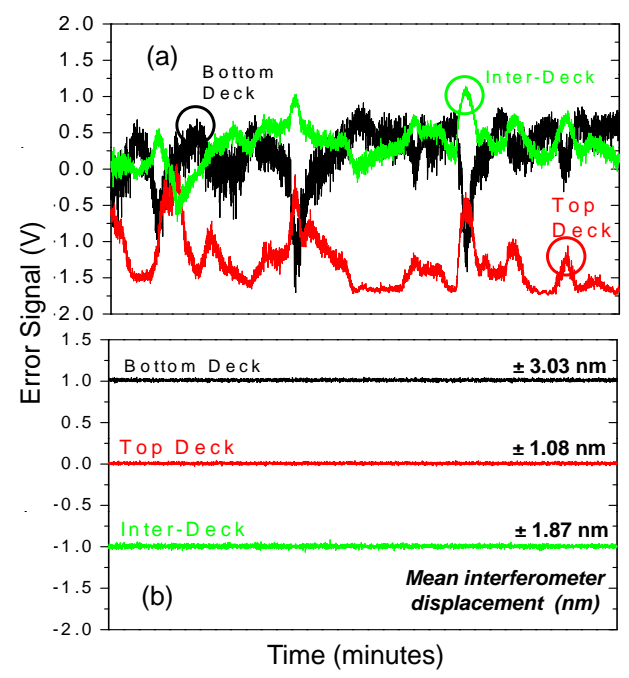

FIG. 5: (Color online) The error signals for the top-deck, bottom-deck and inter-deck interferometers recorded for approximately 10 minutes (a) without and (b) with active stabilization engaged. In (a) circles link each trace to the appropriate label. In (b) the bottom-deck and inter-deck signals are offset from zero for presentation purposes. 


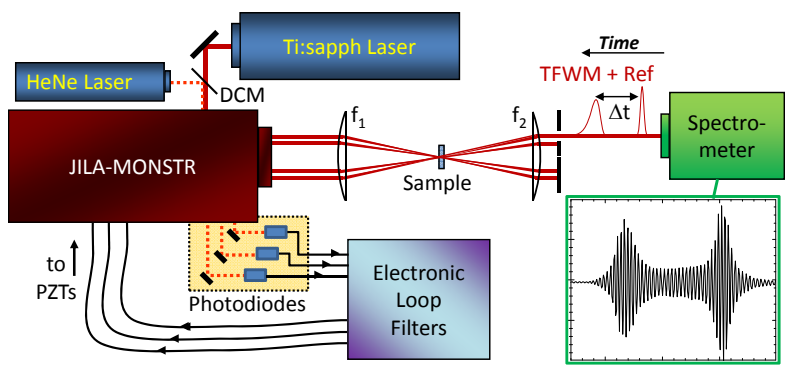

FIG. 6: (Color online) Experimental setup for 2DFT spectroscopy showing the pump and phasestabilization laser, the JILA-MONSTR with diagnostic port, and feedback loops. Also shown is the arrangement of the sample and the collection of light in the spectrometer. A heterodyne reference pulse is used to perform spectral interferometry and reconstruct the time axis t. The inset shows a typical spectral interference pattern recorded for the quantum-well sample. 


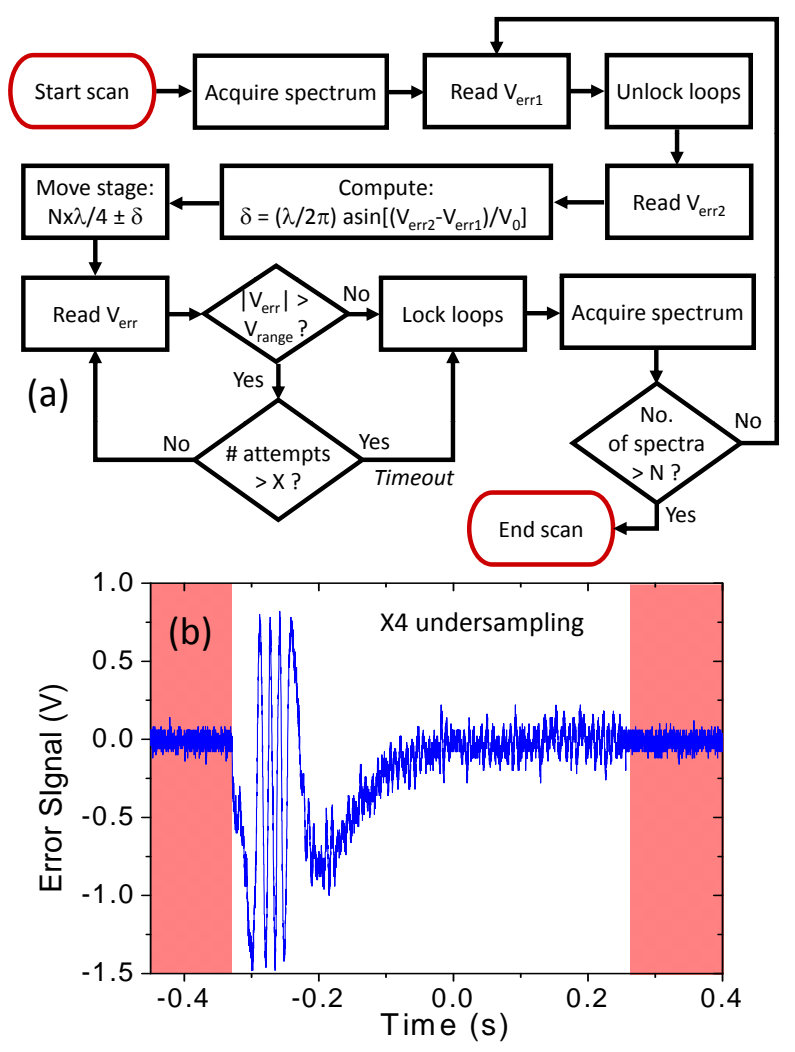

FIG. 7: (Color online) (a) Flow chart of the stepping algorithm to ensure identical steps. There are several measurements of the error signal: $V_{\operatorname{err} 1(2)}$ occur before(after) unlocking the loop filter and $V_{\text {err }}$ is measured after the stage has been moved. $V_{0}$ is half the maximum peak-to-peak error signal, $\mathrm{X}$ is maximum attempts to wait for the error signal to obtain a value within $\mathrm{V}_{\text {range }}$ (near zero), and $\mathrm{N}$ is the total number of spectrum acquired in the 2DFT scan. (b) A typical screen capture of the oscilloscope that monitors the error for the top-deck interferometer while it is being scanned 4 HeNe fringes. The shaded regions mark when the the feedback loop is engaged. 


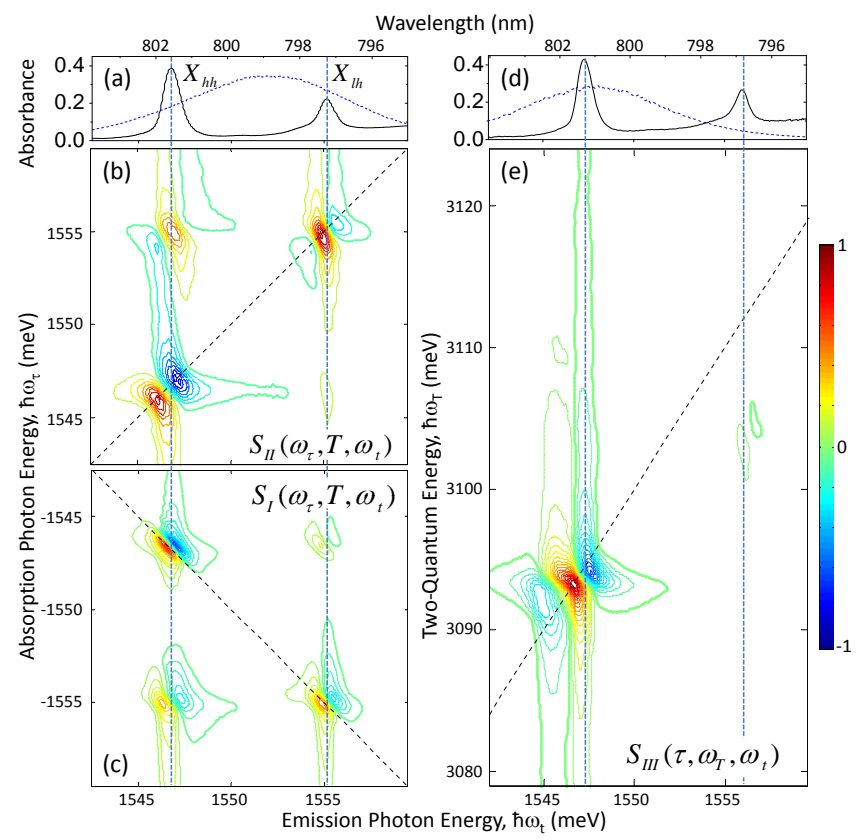

FIG. 8: (Color online) (a,d) Linear absorbance spectrum of the GaAs quantum well sample. Dashed lines show the excitation laser spectra. Two-dimensional Fourier-transform spectra of GaAs quantum wells, showing (b) non-rephasing $\left(S_{I I}\right)$, (c) rephasing $\left(S_{I}\right)$ and (e) two-quantum $S_{I I I}$ techniques. The 2DFT spectra are normalized to the strongest feature, and the thicker contour line encloses the regions of the negative signal. All $2 \mathrm{DFT}$ spectra are for $T=0 \mathrm{fs}$ excitation. The single- (b,c) and two-quantum(e) spectra are acquired for co-circular and cross-circular excitation, respectively. 


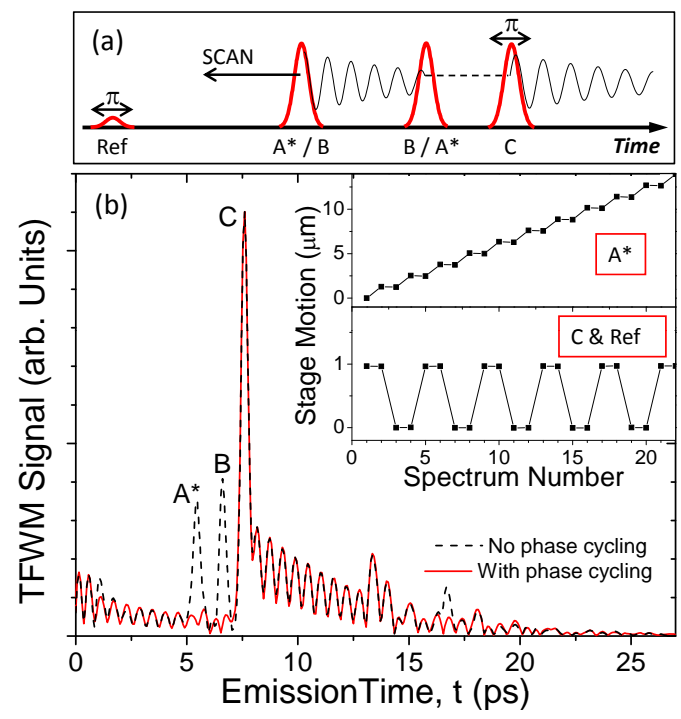

FIG. 9: (Color online) (a) Schematic method of the phase cycling operation for 2DFT spectroscopy. While a time delay is scanned, pulses $\mathrm{C}$ and Ref are simultaneously toggled back and forth by $\pi$ and two spectral interferograms are recorded per data point. (b) Shows the fourier transform of recorded spectral interferograms taken when $T$ and $\tau$ are approximately 1 ps. The dashed line is a result of a single interferogram (i.e. normal operation) and the solid line is for the difference of the two interferograms. The inset shows the stepped slope of the scanned pulse, $\mathrm{A}^{*}$, and the toggled pulses, $\mathrm{C}$ and Ref. 


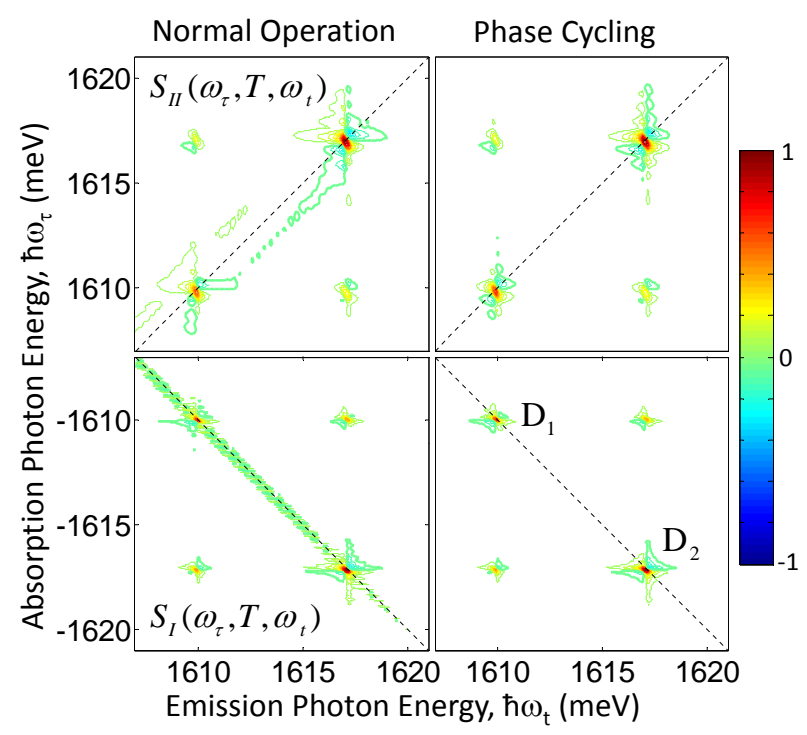

FIG. 10: (Color online) An example of noise reduction in the 2DFT spectra of $\mathrm{K}$ vapor using phase cycling. The left-hand panels show rephasing $\left(S_{I}\right)$ and non-rephasing $\left(S_{I I}\right)$ spectra obtained through normal operation, and the right-hand panels use phase cycling of the $\mathrm{C}$ and Ref pulses by 6 HeNe fringes. 